\title{
Penetrated Shotgun Pellets in Maxillofacial Region: Case Report
}

\author{
Nesrin Saruhan ${ }^{1 *}$, Ümit Ertaş², Mert Ataol², Ergin Öztürk ${ }^{1}$ \\ ${ }^{1}$ Department of Oral and Maxillofacial Surgery, Osmangazi University Faculty of Dentistry, Turkey \\ ${ }^{2}$ Department of Oral and Maxillofacial Surgery, Ataturk University Faculty of Dentistry, Turkey
}

\begin{abstract}
Recently, in many parts of the world, there has been an increase in the number of civilian crimes involving guns and nowadays any oral surgeon in hospital may be confronted with this type of injury. Shotguns are popular worldwide and more of these weapons exist than the rifled types. Shotgun wounds differ from those of other missiles because the spectrum of wound severity is large owing to the fact that the pellets scatter as they travel. In this case report presents a 43-year-old male patient with a large number of pellets in maxillofacial region because of shotgun injury.
\end{abstract}

Keywords: Shotgun, Pellet, Face, Facial Injury.

\section{Introduction}

The number of victims has increased because of firearms has become more widespread in society.[1] Shotgun wounds can be as destructive as high velocity rifles, but they can cause very little injury with longer weaponvictim range.[2] Although there are numerous studies in the literature on injury pattern and treatment algorithms of neck, chest and abdomen injuries, there are few studies on facial injuries because shotgun wounds on the face are relatively uncommon. [1,3] There is no consensus in terms of removing or retaining of shotgun pellets.[4] In this case report, we present a 40 year-old male patient suffering from shotgun wounds on the face, and without any symptoms for 8 years.

\section{Case Report}

A 40-year-old male patient referred to our clinic for routine dental examination. On the radiographic examination, many irregular radiopaque penetrated objects were seen bilaterally. (Figure 1) As a result of detailed anamnesis from the patient, it was learned that the patient suffered from shotgun injury 8 years ago.

According to the patient's information, he was taken to the local hospital after the shotgun injury, and the accessible pellets were removed after basic resuscitation, but other unattainable pellets were left. Then the soft tissue was repaired and the injuries that were exposed were closed. The maxillofacial complex had still a large number of pellets, but functional failure was not present and no procedure was considered due to difficult access and a close anatomical relationship with the pellet and the vital structures.

\section{Discussion}

Shotgun wounds to the maxillofacial region which are uncommon can result from suicide, homicide or accident. It can cause destructive functional and aesthetic consequences for patients. [5] The most patients die because of these injuries, but some can survive with serious injuries on their face.[6]

There are two forms of injury originating from the pellets: minor which is required care in the emergency department, and serious which is required admission to the hospital and frequently surgery intervention. Serious injuries sometimes lead to long-term functional disability. [7] Although many shotgun pellets were lodged into maxillofacial region, none of them did not destroy the vital structures; therefore patient has survived so far.

Sherman [8] suggested a classification scheme based on the distance from the shotgun to the victim for shotgun injuries. The close-range shotgun injuries cause severe soft tissue loss such as bone fractures and intracranial penetration because of significant amounts of kinetic energy to the face.

The treatment of shotgun injuries seen on the maxillofacial region may be extremely difficult, and it requires individual treatment because of complex nature. The treatment options of shotgun injuries include conservative approach of debridement and immediate reconstruction. The principles of conservative debridement, extensive wound cleanup, and the primary replacement of soft tissue defects will lead to better functional and aesthetic restoration in a shorter time.[9] In our case report, the pellets were left because of the absence of any clinical symptoms. 


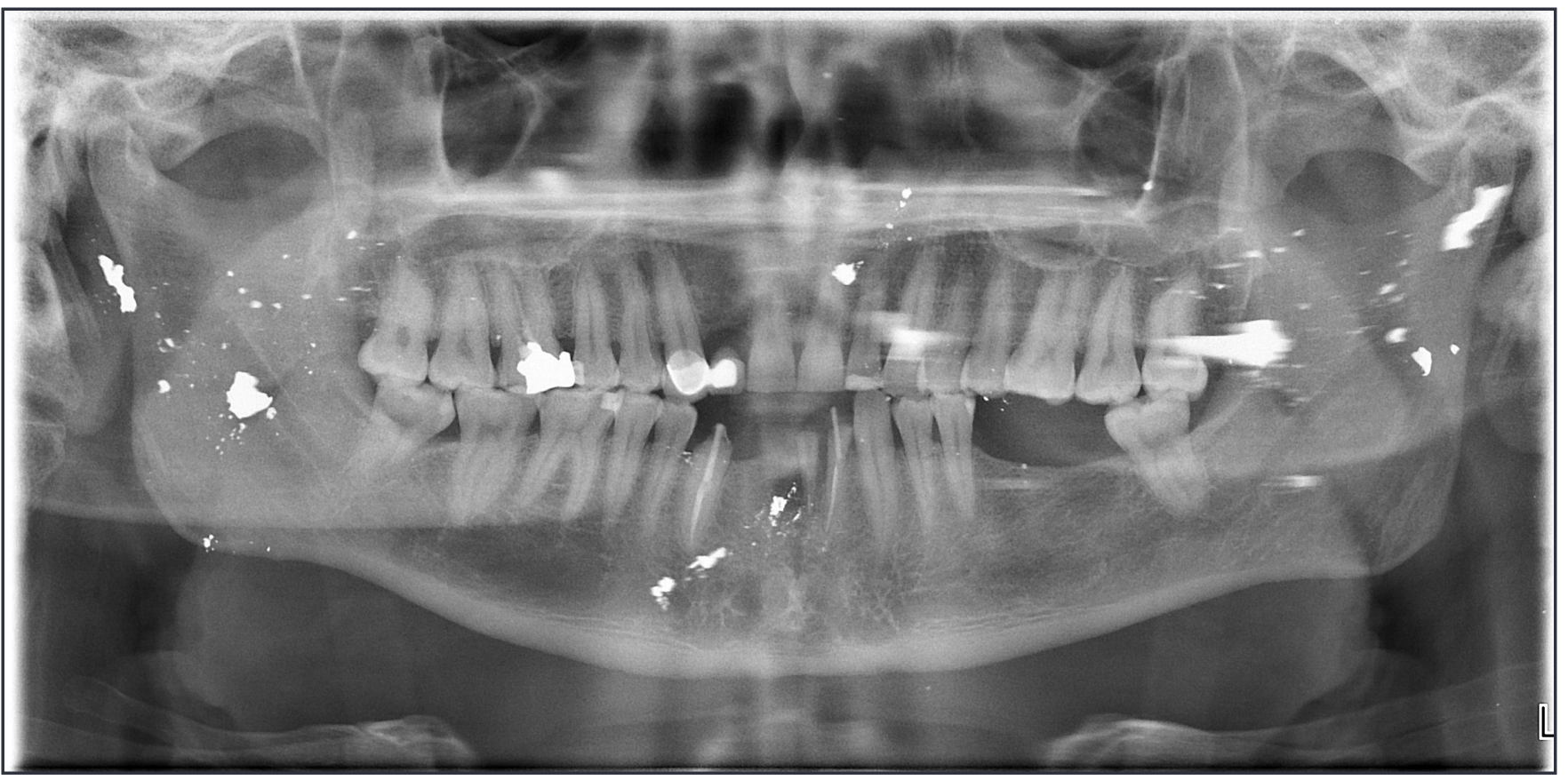

Fig. 1: Large number of pellets in maxillofacial region was seen in panoramic radiography.

As a result, the pellets can remain in the maxillofacial region for many years without any obvious symptoms and can be left in situ without any intervention.

\section{Reference}

1. Chen, A.Y., M.G. Stewart, and G. Raup, Penetrating injuries of the face. Otolaryngology--head and neck surgery : official journal of American Academy of Otolaryngology-Head and Neck Surgery, 1996. 115(5): p. 464-70.

2. Ordog, G.J., J. Wasserberger, and S. Balasubramaniam, Shotgun wound ballistics. The Journal of trauma, 1988. 28(5): p. 624-31.

3. Arslan, H., et al., Problem fractures associated with gunshot wounds in children. Injury, 2002. 33(9): p. 743-9.

4. Kara, M.I., H.B. Polat, and S. Ay, Penetrated shotgun pellets: a case report. European journal of dentistry, 2008. 2(1): p. 59-62.
5. Vayvada, H., et al., Management of close-range, highenergy shotgun and rifle wounds to the face. The Journal of craniofacial surgery, 2005. 16(5): p. 794-804.

6. Desinan, L. and G.M. Mazzolo, Gunshot fatalities: suicide, homicide or accident? A series of 48 cases. Forensic science international, 2005. 147 Suppl: p. S37-40.

7. Scribano, P.V., et al., Pediatric nonpowder firearm injuries: outcomes in an urban pediatric setting. Pediatrics, 1997. 100(4): p. E5.

8. Sherman, R.T. and R.A. Parrish, Management of shotgun injuries: a review of 152 cases. Journal of Trauma and Acute Care Surgery, 1963. 3(1): p. 76-86.

9. Goodstein, W.A., A. Stryker, and L.J. Weiner, Primary treatment of shotgun injuries to the face. The Journal of trauma, 1979. 19(12): p. 961-4.

*Corresponding author:

Nesrin SARUHAN, Department of Oral and Maxillofacial Surgery, Osmangazi University Faculty of Dentistry, Eskişehir, TURKEY.

Phone: +9190442 23118 01, Fax: +90 4422361375 ,

Email: dt_nesrin@yahoo.com

Financial or other Competing Interests: None. 